AGSAPPLIED MATERIALCaltech Library

Subscriber access provided by Caltech Library

\title{
Article
}

\section{Shear-Induced Brittle Failure along Grain Boundaries in Boron Carbide}

Xiaokun Yang, Shawn P Coleman, Jerry Lasalvia, William A. Goddard, and Qi An

ACS Appl. Mater. Interfaces, Just Accepted Manuscript • DOI: 10.1021/acsami.7b16782 • Publication Date (Web): 18 Jan 2018

Downloaded from http://pubs.acs.org on January 19, 2018

\section{Just Accepted}

"Just Accepted" manuscripts have been peer-reviewed and accepted for publication. They are posted online prior to technical editing, formatting for publication and author proofing. The American Chemical Society provides "Just Accepted" as a free service to the research community to expedite the dissemination of scientific material as soon as possible after acceptance. "Just Accepted" manuscripts appear in full in PDF format accompanied by an HTML abstract. "Just Accepted" manuscripts have been fully peer reviewed, but should not be considered the official version of record. They are accessible to all readers and citable by the Digital Object Identifier (DOI®). "Just Accepted" is an optional service offered to authors. Therefore, the "Just Accepted" Web site may not include all articles that will be published in the journal. After a manuscript is technically edited and formatted, it will be removed from the "Just Accepted" Web site and published as an ASAP article. Note that technical editing may introduce minor changes to the manuscript text and/or graphics which could affect content, and all legal disclaimers and ethical guidelines that apply to the journal pertain. ACS cannot be held responsible for errors or consequences arising from the use of information contained in these "Just Accepted" manuscripts. 


\title{
Shear-Induced Brittle Failure along Grain Boundaries in Boron Carbide
}

\author{
Xiaokun Yang ${ }^{1}$, Shawn P. Coleman ${ }^{2}$, Jerry Lasalvia ${ }^{2}$, William A. Goddard III $^{3}$ and Qi An ${ }^{1, *}$ \\ ${ }^{1}$ Department of Chemical and Materials Engineering, University of Nevada, Reno, \\ Reno, Nevada, 89577, United States \\ ${ }^{2}$ United States Army Research Laboratory, Aberdeen Proving Ground, \\ Aberdeen, MD 21005, United States \\ ${ }^{3}$ Materials and Process Simulation Center, California Institute of Technology, \\ Pasadena, CA 91125, United States \\ *Corresponding author E-mail: qia@unr.edu
}

\begin{abstract}
The role that grain boundaries (GB) can play on mechanical properties has been studied extensively for metals and alloys. However, for covalent solids such as boron carbide $\left(\mathrm{B}_{4} \mathrm{C}\right)$, the role of $\mathrm{GB}$ on the inelastic response to applied stresses is not well established. We consider here the unusual ceramic, boron carbide $\left(\mathrm{B}_{4} \mathrm{C}\right)$, which is very hard and lightweight but exhibits brittle impact behavior. We used quantum mechanics (QM) simulations to examine the mechanical response in atomistic structures that model $\mathrm{GBs}$ in $\mathrm{B}_{4} \mathrm{C}$ under pure shear and also with biaxial shear deformation that mimics indentation stress conditions. We carried out these studies for two simple GB models including also the effect of adding Fe atoms (possible sintering aid and/or impurity) to the GB. We found that the critical shear stresses of these GB models are much lower than for crystalline and twinned $\mathrm{B}_{4} \mathrm{C}$. The two GB models lead to different interfacial energies. The higher interfacial energy at the GB only slightly decreases the critical shear stress but dramatically increases the critical failure strain. Doping the GB with Fe decreases the critical shear stress of at the boundary by $14 \%$ under pure shear deformation. In all GBs studied here, failure arises from deconstructing the icosahedra within the GB region under shear deformation. We find that Fe dopant interacts with icosahedra at the GB to facilitate this deconstruction of icosahedra. These results provide significant insight for designing polycrystalline $\mathrm{B}_{4} \mathrm{C}$ with improved strength and ductility.
\end{abstract}

Keywords: Boron carbide, Grain boundaries, DFT, Impurity effect, Deformation mechanism 


\section{Introduction}

The excellent properties of $\mathrm{B}_{4} \mathrm{C}$ (such as high melting temperature, high thermal stability, high hardness, high abrasion resistance, low density, excellent neutron absorption and relatively low cost) make it a promising candidate for applications in body armors, abrasive grits, wearresistance components, and absorbent nuclear materials. ${ }^{1-10}$ However, engineering applications of $\mathrm{B}_{4} \mathrm{C}$ to body armor or abrasive powders have been impeded by the abnormal brittle failure under high pressure due to amorphous shear band formation. ${ }^{5,10,11}$ Amorphous bands had also been observed in both simulated shear and scratch experiments, ${ }^{11-14}$ suggesting that it is a major failure mechanism in $\mathrm{B}_{4} \mathrm{C}$, but it is not known what role is played by grain boundaries (GBs).

Characterizing how the atomistic structure at GBs affects the mechanical properties is essential to guiding synthetic processes [hot-pressing (HP) or spark plasma sintering (SPS)] to tailor the materials properties of boron carbide $\left(\mathrm{B}_{4} \mathrm{C}\right) .{ }^{15-18}$ Experimental studies of $\mathrm{B}_{4} \mathrm{C}$ using transmission electron microscopy (TEM) revealed both relatively clean GBs and second phase inclusions (such as graphite, $\mathrm{Al}_{2} \mathrm{O}_{3}, \mathrm{Fe}_{2} \mathrm{O}_{3}$, etc.). ${ }^{17-19}$ It has been speculated that the nature of these GBs and of dopant additives at the GBs can affect strongly the strength, ductility, dynamic mechanical properties, and ballistic performances of $\mathrm{B}_{4} \mathrm{C} .{ }^{18,19}$ However, little is known about the atomistic structures and mechanical response at the GBs.

To explain the abnormal brittle failure of $\mathrm{B}_{4} \mathrm{C}$, large-scale reactive force field (ReaxFF) reactive molecule dynamics simulations were performed to examine shear deformation for systems with cells lengths of $\sim 25 \mathrm{~nm}$ ( $\sim 200,000$ atoms), observing formation of $\sim 3 \mathrm{~nm}$ wide amorphous shear bands for shear along both of the two easiest slip systems: $(001) /<100>$ and $(111) /<2 \overline{1} \overline{1}>.^{20}$ In this earlier paper we showed that amorphous shear bands form because as 
planes of icosahedral clusters are sheared to break $\mathrm{C}-\mathrm{B}$ intercluster bonds, they then react with the $\mathrm{B}$ of the $\mathrm{C}-\mathrm{B}-\mathrm{C}$ chains as they tilt over during the shear. ${ }^{10}$ However, no studies have yet been on polycrystalline $\mathrm{B}_{4} \mathrm{C}$, to determine how $\mathrm{GBs}$ might affect the amorphous shear band formation and abnormal brittle failure.

The ground state structure of $\mathrm{B}_{4} \mathrm{C}$ is composed of two main structural units: the 12-atom $\mathrm{B}_{11} \mathrm{C}_{\mathrm{p}}$ icosahedral cluster in which the $\mathrm{C}$ is at the polar sites $\mathrm{C}_{\mathrm{p}}$ connecting to other icosahedra plus the 3 -atom $\mathrm{C}-\mathrm{B}-\mathrm{C}$ chains aligned along the $<111>$ direction. ${ }^{10}$ The middle $\mathrm{B}$ in the $\mathrm{C}-\mathrm{B}-\mathrm{C}$ chain donates one extra electron to $\mathrm{B}_{11} \mathrm{C}_{\mathrm{p}}$ icosahedron leading to 13 strong multicenter intraicosahedral bonds (Wade's rule). ${ }^{21}$ These strong covalent bonds in $\mathrm{B}_{4} \mathrm{C}$ result in low selfdiffusion and poor sinterability. Even so, sharp clean GBs have been observed experimentally. Irrespective of grain orientation ${ }^{18,19}, \mathrm{GBs}$ in $\mathrm{B}_{4} \mathrm{C}$ are structurally sharp and free of glassy oxide nanolayer films typically observed in other ceramics. ${ }^{17-19}$ Because the structure and chemistry of GBs have been shown to have a strong impact on the densification, microstructures, and mechanical properties of other ceramics such as silicon carbide $(\mathrm{SiC})$ and silicon nitride $\left(\mathrm{Si}_{3} \mathrm{~N}_{4}\right)$, it is critical to understand the atomic structures within GBs regions. ${ }^{22,23}$

$\mathrm{B}_{4} \mathrm{C}$ bodies are produced from powders that are densified by pressure-assisted sintering techniques such as hot-pressing or spark-plasma sintering. ${ }^{24-27}$ Metallic impurities are common in $\mathrm{B}_{4} \mathrm{C}$ because the powders are produced by grinding and milling $\mathrm{B}_{4} \mathrm{C}$ "ingots" (formed by reacting $\mathrm{B}_{2} \mathrm{O}_{3}$ and $\mathrm{C}$ in an arc-melting furnace) using steel implements. ${ }^{27}$ Even with acid washing, metallic impurities remain. For example, TEM energy-dispersive X-ray spectroscopy (EDS) analysis of commercial hot-pressed $\mathrm{B}_{4} \mathrm{C}$ ceramics showed detectable amounts of $\mathrm{Fe}, \mathrm{Al}, \mathrm{Si}, \mathrm{V}, \mathrm{Ti}$, $\mathrm{Cr}$, and $\mathrm{Mo}$ in addition to $\mathrm{B}$ and $\mathrm{C}^{6}$ In addition, the higher impurity level can further reduce mechanical properties such as fracture toughness, flexural strength, and hardness which can 
degrade performance in such applications such as ballistic protection. ${ }^{15}$ On the other hand, fundamental knowledge on the distribution and existence of the reaction products from the additives and impurities in $\mathrm{B}_{4} \mathrm{C}$ is essential to understand their potential positive influence on these mechanical properties, especially on amorphization and brittle failure, leading to the development of new boron carbide ceramics with improved performance. Therefore, it is also essential to understand what effect grain boundaries and impurities may have on the mechanisms governing the amorphization and brittle failure of $\mathrm{B}_{4} \mathrm{C}$.

The integrity of icosahedral clusters plays an important role in determining the mechanical failure of $\mathrm{B}_{4} \mathrm{C}$ because the amorphous shear bands initiate from deconstructing the icosahedral cluster. ${ }^{10}$ In this article, we examined two types of GB models

- GB-I (111)/(高3) in which integral icosahedral clusters are along GBs

- GB-II $(2 \overline{1} \overline{1}) /(\overline{2} 11)$ in which the deconstructed icosahedral clusters are along GBs

as representative general interface structures. Then, the Fe atoms were doped into the GB-I model to illustrate the impurity effects. We use Fe as a representative element since it had been reported as a major and high concentration impurity that observed in commercial $\mathrm{B}_{4} \mathrm{C}$ and crystalline boron powders. ${ }^{28}$ In addition, $\mathrm{B}_{4} \mathrm{C}$ is usually experimentally synthesized using steel implements. ${ }^{24-27}$ Finally, we used quantum mechanics (QM) simulations to examine the mechanical response of all three GB models under both pure shear and biaxial shear deformation. Our simulations reveal active deformation mechanisms within the GB models that are starkly different than crystalline $\mathrm{B}_{4} \mathrm{C}$. We find that the critical shear strength for GB-I and GB-II models are 25.5 and $23.7 \mathrm{GPa}$, respectively, which are much less than for crystalline (38.9 GPa) and twinned $\mathrm{B}_{4} \mathrm{C}(43.6 \mathrm{GPa}) \cdot{ }^{10,29}$ This suggests that existence of GBs, dramatically decrease the strength of $\mathrm{B}_{4} \mathrm{C}$. We find that brittle failure of $\mathrm{GB}$ structures under pure shear deformation arises 
from shear-induced icosahedra disintegration in the GB region, while brittle failure under biaxial shear deformation starts from compressing the icosahedral layers in GB region. Doping Fe atoms into the GB-I model leads to a negative enthalpy of formation (referenced to $\alpha$-Fe and the GB-I model) suggesting that impurities preferred to the GBs regions. We find that shearing the Fedoped GB model leads to a further decrease in the critical shear stress of GB by $3.5 \mathrm{GPa}(14 \%)$ due to the interaction of Fe atoms with icosahedral clusters in the GB.

\section{Computational Methodology}

All DFT calculations were performed with the VASP package, ${ }^{30-32}$ using the Perdew-BurkeErnzerhof (PBE) functional ${ }^{33}$ and the projector augmented wave (PAW) method to account for the core-valence interactions. ${ }^{34}$ The energy cutoff for the plane wave expansion was $600 \mathrm{eV}$ and Brillouin zone integration was performed on $\Gamma$-centered symmetry-reduced Monkhorst-Pack meshes with a fine resolution of $2 \pi \times 1 / 40 \AA^{-1}$ for all calculations except for both pure shear and biaxial shear deformation. The energy error for terminating electronic self-consistent field (SCF) and the force criterion for the geometry optimization were set equal to $10^{-6} \mathrm{eV}$ and $10^{-3} \mathrm{eV} / \AA$, respectively.

For undoped GB models, the electron partial occupancies were determined using the tetrahedron method with Blöchl corrections. ${ }^{35}$ While, the Methfessel-Paxton scheme ${ }^{36}$ was applied to determine the electron partial occupancies for the Fe-doped GB model.

To determine the critical shear strength under pure shear deformation, we imposed the shear strain along the GB planes while allowing full structural relaxation of other five strain components. ${ }^{37} \mathrm{~A} 1 \%$ level of strain was predefined as the small strain increment for each 
deformation step. The stress is defined as the force per deformed area, and the strain is defined as the true strain.

To simulate the mechanical response under indentation experiments, we applied biaxial shear deformation on the GBs structures, adjusted to mimic the deformation under the indenter by imposing the relations $\sigma_{\mathrm{zz}}=\sigma_{\mathrm{zx}} \times \tan \Phi$ where $\sigma_{\mathrm{zz}}$ is the normal stress, $\sigma_{\mathrm{zx}}$ is the shear stress and $\Phi$ is the centerline-to face angle of the indenter $\left(\Phi=68^{\circ}\right.$ for Vickers indenter $){ }^{38}$ The other four strain components were relaxed in the biaxial shear deformation. The residual stresses after relaxing were less than $0.2 \mathrm{GPa}$ for both pure shear and biaxial shear deformation. A more approximate $2 \times 2 \times 2 \mathrm{k}$-point grid mesh in the Brillouin zone was applied in both pure shear and biaxial shear deformation simulations.

The force criterion for geometry optimization is $1.0 \times 10^{-3} \mathrm{eV} / \AA$. Considering that the area in atomic simulations is $\sim \AA^{2}$, the uncertainty for the stress should be less than $1.0 \times 10^{-3} \mathrm{eV} / \AA / \AA^{2}=$ $\sim 0.16 \mathrm{GPa}$. Therefore, we used one significant digit for the stress. The energy convergence criterion is $10^{-6} \mathrm{eV}$. Thus, the uncertainty for the interfacial energy should be $10^{-6} \mathrm{eV} / \AA^{2}=\sim 0.01$ $\mathrm{mJ} / \mathrm{m}^{2}$. Here, we used one significant digit for the interfacial energy. For the lattice parameters and densities, we used three significant digits since the uncertainty for atomic positions is less than $10^{-6} \AA$.

\section{Results and Discussion}

\subsection{Atomistic structure of GB models}

We constructed GB models based on $\left(\mathrm{B}_{11} \mathrm{C}_{\mathrm{p}}\right) \mathrm{CBC}$ configuration since it is the ground state structure for stoichiometry $\mathrm{B}_{4} \mathrm{C}$. The GB structures in $\mathrm{B}_{4} \mathrm{C}$ are very complex. Although the grain orientations can be determined using electron backscatter diffraction (EBSD), there is little 

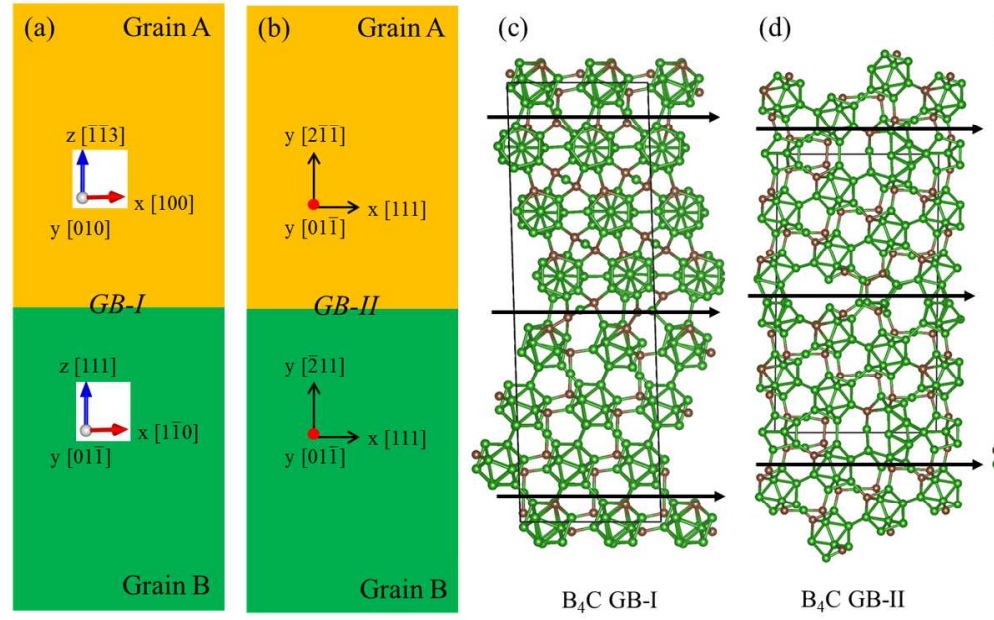

(e)

Figure 1. The schematic $(a, b)$ models and relaxed DFT structures(c-e) for $B_{4} C$ GB-I and GB-II models. (c) $B_{4} C G B-I$ (d) $B_{4} C$ model GB-II model, and (e) Fe-doped $B_{4} C$ GB structure. The boron and carbon atoms are represented by the green and sienna balls, respectively. The Fe atom is represented by the yellow ball.

Two grain boundaries (GB1 and GB2) are contained in each GB model because of the periodic boundary conditions. In the GB-I model, the $\mathrm{B}_{4} \mathrm{C}$ structure in Grain A has its [111] C-B$\mathrm{C}$ chain perpendicular to the GB, whereas, the [111] C-B-C chain forms an angle $\sim 35^{\circ}$ with the GB in Grain B. To accommodate the lattice mismatch, the C-B-C chain forms an angle $\sim 35^{\circ}$ to 
GB, like a twinned chain structure in Grain A (Fig. 1(c)). We keep the C-B-C chain perpendicular to the GB in the Grain B (Fig. 1(c)). The GB-I structure was relaxed by DFT simulation, leading to equilibrium supercell lattice parameters of $\mathrm{a}=10.921 \AA, \mathrm{b}=5.313 \AA, \mathrm{c}=$ $30.319 \AA, \alpha=90.4^{\circ}, \beta=91.7^{\circ}$, and $\gamma=63.0^{\circ}$ with a density of $\rho=2.460 \mathrm{~g} / \mathrm{cm}^{3}$. It is interesting to note that the icosahedra stay intact along the GBs in the GB-I model. Since GB1 is not identical to GB2, we computed the average GB interfacial energy of GB-I model by referencing to bulk $\left(B_{11} C_{p}\right) C B C$. This leads to an average interfacial energy of $4729.2 \mathrm{~mJ} / \mathrm{m}^{2}$ for GB-I model.

The GB-II model represents a more general $\mathrm{B}_{4} \mathrm{C}$ boundary. In the GB-II model, the icosahedra must be deconstructed along the GBs to accommodate the lattice mismatch, as shown in Fig. 1(d). The structure was relaxed using DFT, to obtain equilibrium supercell lattice parameters of a $=12.172 \AA, b=5.530 \AA, c=20.975 \AA, \alpha=89.5^{\circ}, \beta=89.5^{\circ}$, and $\gamma=90.7^{\circ}$ with a density of $\rho=$ $2.429 \mathrm{~g} / \mathrm{cm}^{3}$. Since the two GBs are not identical in the GB-II model, the computed average interfacial energy for GB-II model is $5345.5 \mathrm{~mJ} / \mathrm{m}^{2}$, which is $10 \%$ larger than that of GB-I model. The GB-II model has $151 \mathrm{~B}$ and $36 \mathrm{C}$ atoms. Therefore, we calculate the interfacial energy using bulk $\left(B_{11} C_{p}\right) C B C$ as the reference to $B_{144} C_{36}$ and $\alpha-B_{12}$ as the reference for the additional $7 \mathrm{~B}$ atoms.

To examine the impurity effect on GB properties and failure mechanisms, we constructed a Fe-doped grain boundary model based on GB-I model. The Fe impurity is found to be from $0.1 \sim 1 \mathrm{wt} \%$ in experiments. ${ }^{28}$ In our GB model, we inserted two Fe atoms into the GB-I model with 210 atoms, leading to $4.6 \mathrm{wt} \%(0.94 \mathrm{at} \%)$ of Fe impurity in $\mathrm{B}_{4} \mathrm{C}$, as shown in Fig. 1(e). In order to find the most plausible positions for Fe atoms in GB region, we computed the atomic volume in GB-I model by constructing the Voronoi polyhedral for each atom. ${ }^{40}$ Then the Fe atoms were placed in the middle of two B atoms that have the largest atomic volume among 
chain B and icosahedral B atoms, respectively. Then the structure is optimized using DFT simulations. After optimization the Fe was positioned at free space of GB1 with a distance of $2.357 \AA$ to the $\mathrm{B}$ atom in the nearest C-B-C chain, and $2.985 \AA$ and $2.269 \AA$ to the nearest polar B atom in Grain A and Grain B, respectively. This Fe-doping leads to a negative of enthalpy of formation of $-0.71 \mathrm{eV} /$ supercell referenced to $\alpha$-Fe and GB-I model, suggesting that Fe-doping stabilizes the GB. This suggests that the impurity elements prefer to be located within the GB region in polycrystalline $\mathrm{B}_{4} \mathrm{C}$. The negative formation energy suggests that $\mathrm{Fe}$ likes to be at the GB interface. This is consistent with the B-C-Fe phase diagram ${ }^{41}$ that the $\mathrm{Fe}$ is involved in the ternary phase when the $\mathrm{Fe}$ at. $\%$ is above $40 \%$.

\subsection{Mechanical response of GBs in $\mathrm{B}_{4} \mathrm{C}$}

\subsubsection{Deformation mechanisms under pure shear}

The deformation mechanism of boron carbides ${ }^{42-45}$ and other ceramics has been widely investigated. ${ }^{46}$ In particular, strain-stiffening has been observed in $\mathrm{Fe}_{3} \mathrm{C}$ and $\mathrm{Al}_{3} \mathrm{BC}_{3}$ ceramics using the QM simulations. ${ }^{46}$ To examine the mechanical response of $\mathrm{B}_{4} \mathrm{C}$ GBs under pure shear deformation, we applied finite shear deformation on the three GB models in steps of $2 \%$ strain until failure. Shear was induced in the x-direction of the simulation cell, which corresponds to the [100] direction for Grain A and [11̄0] for Grain B in the GB-I and Fe-doped GB models. The GB-II model was sheared along [111] direction in both grains. The shear stress-strain relationships for these GB models are displayed in Fig. 2. The GB-I model deforms elastically to 0.173 strain, while the GB-II model deviates from the elastic deformation at 0.040 strain because of the disintegrated icosahedra within the GB regions. The Fe impurity in the GB promotes deviations from elastic deformation at 0.061 strain, which is $65 \%$ lower than that of the undoped GB-I model. The critical shear stresses for GB-I, GB-II and Fe-doped GB models are 25.5, 23.7, 
and $22.0 \mathrm{GPa}$, respectively. These values are much lower than those of crystalline $(38.9)^{10}$ and nanotwinned $\mathrm{B}_{4} \mathrm{C}$ (43.6). ${ }^{29}$ The strength of the GB-I model is higher than the GB-II model, which correlates with the lower GB interfacial energy of GB-I. However, the critical failure strain for GB-II is 0.276 , which is $45 \%$ larger than that of GB-I model (0.191), suggesting that the GB-II model is more ductile than GB-I model. Since the GB-II model is only $7.1 \%$ lower in strength, but $45 \%$ higher in critical failure strain (compared to the GB-I model), a critical design strategy to improve the ductility of polycrystalline $\mathrm{B}_{4} \mathrm{C}$ could be to increase the interfacial energy of the present GBs. Since Fe impurities decrease the critical shear stress without significantly changing the failure strain, it is important to avoid the metal impurities to improve the mechanical properties of polycrystalline $\mathrm{B}_{4} \mathrm{C}$.

Our previous QM study examined 11 possible slip systems to find that ideal shear stress for bulk single-crystal $\mathrm{B}_{4} \mathrm{C}$ is $38.9 \mathrm{GPa}^{10}$ Since the critical stresses shearing along the GBs in this work are $34.5 \%$ and $39.1 \%$ lower than this ideal shear stress, mechanical failure would likely initiate from GB regions. In addition, since the presence of the Fe impurities further reduces the critical shear strength of the GB structure, we expect that the mechanical failure likely initiates from impurity atoms contained in the GB regions. Although the GB regions are much weaker compared to the single-crystalline $\mathrm{B}_{4} \mathrm{C}$, transgranular failure is observed experimentally to be a major failure mode in polycrystalline $\mathrm{B}_{4} \mathrm{C} .^{47,48}$ This is due to failure being governed by the initiation of cracks from large second phase inclusions (introduced during processing) by tensile stresses. 


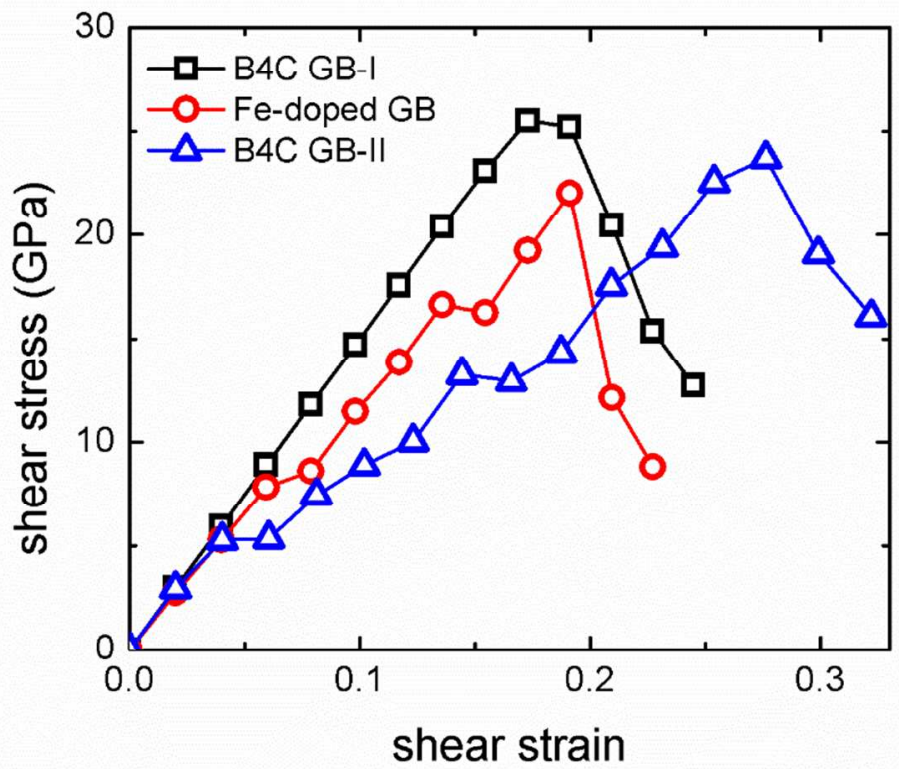

Figure 2. Shear-stress-shear-strain relationship of $B_{4} C$ GB-I model (black $\square$ ), $F e-B_{4} C G B$ (red $\circ)$ and $B_{4} C$ GB-II model (blue $\Delta$ ) structures shearing along the GBs under pure shear deformation.

To understand the atomic mechanisms of failure at the GB under pure shear, we studied the trajectories of the GB-I model, as shown in Fig. 3(a)-(h). The intact structure at 0 strain is displayed in Fig. 3(a). Initially, the GB-I model shears elastically to 0.173 strain, corresponding to its maximum shear stress. No icosahedra are disintegrated, as shown in Fig. 3(b). As the shear strain increases to 0.209 , an obvious deconstruction is observed in the icosahedral clusters in GB1, as shown in Fig. 3(c). However, the icosahedra within GB2 are not disintegrated, suggesting that GB2 has better shear resistance than GB1. As the shear strain further increases to 0.245, the icosahedra in GB1 are fully deconstructed, but the icosahedra in GB2 are still not disintegrated, as shown in Fig. 3(d). Therefore, in the GB-I model, GB1 is weaker than GB2 under shear deformation, which promotes the initiation of failure from the GB1 region.

In order to examine the detailed failure process, we magnify the atomic structures within GB1 area under pure shear deformation, shown in Fig. 3(e)-(h). Initially, the angle of B166-C39-B155 
significantly increases from $71.9^{\circ}$ to $106.1^{\circ}$ from 0 to 0.173 strain, breaking the B166-B155 bond within the icosahedron with the bond distance increasing from 2.051 to $2.850 \AA$ (Fig. 3(e), (f)). As shear strain increases to 0.209 , the icosahedra deconstruct due to the interaction with nearby icosahedron (Fig. 3(g)). The nearby icosahedron also deconstruct with the B111 and B118 atoms being kicked out from the icosahedron and the distance between B166 and B155 further increasing to $4.933 \AA$. The angle of $\mathrm{C} 2-\mathrm{B} 14-\mathrm{C} 3$ chain decreases from $174.6^{\circ}$ to $134.9^{\circ}$. As the shear strain continuously increases to 0.245 strain, the polar carbon atom C39 and other B atoms (e.g. B155) interact with neighbor icosahedra, leading to the full deconstruction of one layer of icosahedra in GB1, leaving a bent angle of $128.0^{\circ}$ for C2-B14-C3 chain. The evolution of the C2-B14-C3 angle and the B166-B155 bond distance are displayed in Fig. S1 of the Supporting Information (SI).

(a)

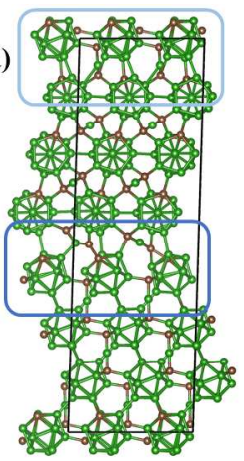

Strain $=0$

(e)

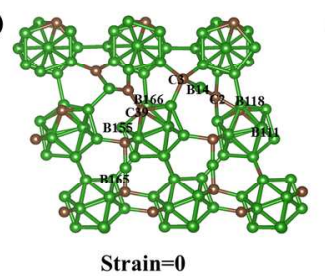

(b)

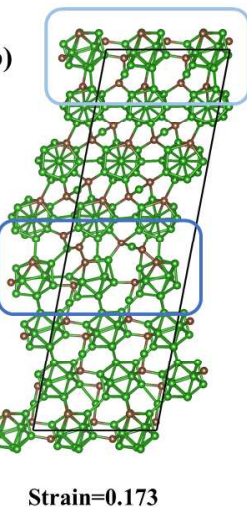

(f)

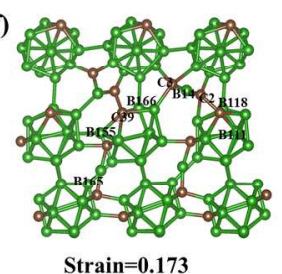

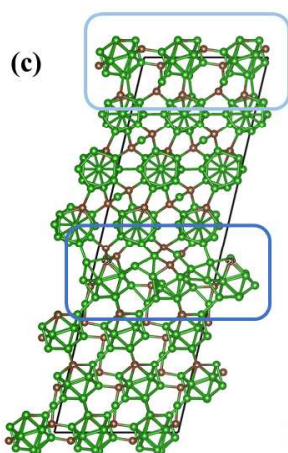

Strain=0.209

(g)

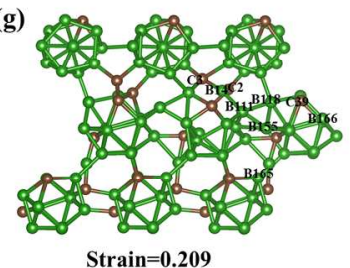

(d)

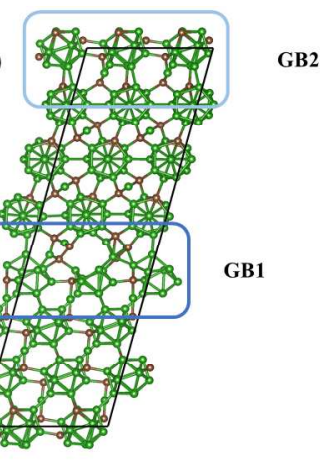

Strain $=\mathbf{0 . 2 4 5}$

\section{(h)}

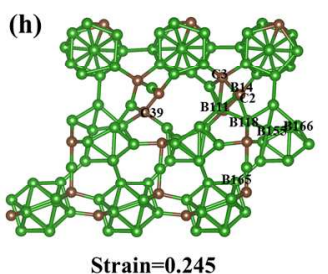

Figure 3. The structures evolution of GB-I model under pure shear deformation: (a,e) the intact structure; $(b, f)$ the structure at 0.173 strain corresponding to the maximum shear stress; $(c, g)$ the structure at 0.209 strain corresponding to the initial failure step; $(d, h)$ the fully deconstructed GB1 structure at 0.245 strain. The boron and carbon atoms are represented by the green and sienna balls, respectively. 
To understand the failure mechanisms of the GB-II model, we extracted the evolution of atomistic structures under various shear strains, as shown in Fig. 4. The structure at 0 strain is displayed in Fig. 4(a). As the shear strain increases to 0.276 corresponding to the maximum shear stress, atoms B14 and B81 form a new bond, as shown in Fig. 4(b). Then, the B3-B7 bond distance increases from 1.773 at 0.276 strain to $3.838 \AA$ at 0.299 strain and breaks as shear strain increases, leading to the separation of fused-icosahedra in the GB region, as shown in Fig. 4(c). This leads to the mechanical failure of the GB-II model.

(a)

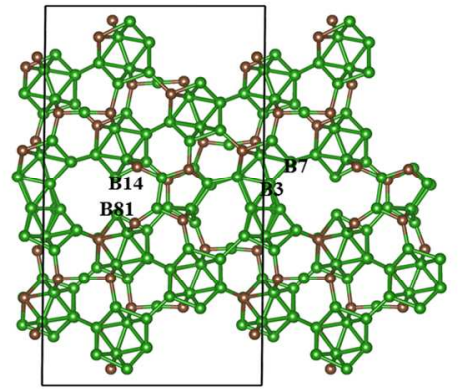

Strain $=0$

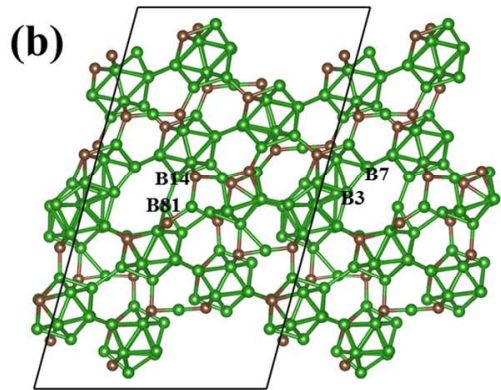

Strain $=\mathbf{0 . 2 7 6}$

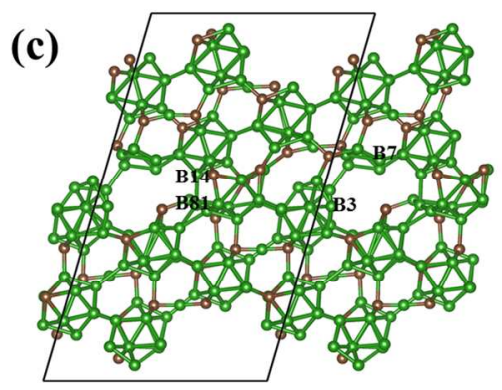

Strain $=\mathbf{0 . 2 9 9}$

Figure 4. The structures evolution of GB-II model: (a) the intact structure; (b) the structure at 0.276 strain corresponding to the maximum shear stress; (c) the failed structure at 0.299 strain. The boron and carbon atoms are represented by the green and sienna balls, respectively.

To examine how an Fe impurity affects the failure mechanism, we examined the structure evolution of the Fe-doped GB model, as shown in Fig.5. The whole simulation cells are displayed in Fig. 5(a-c) while the failure regions are enlarged in Fig. 5(d-f). At 0 strain, the icosahedral B118 atom has been kicked out the icosahedron to accommodate the presence of Fe atom. But the icosahedron does not disintegrate, as shown in Fig. 5(a,d). Compared to the GB-I model, at 0 strain the B118-B111-B105 angle increases from $59.5^{\circ}$ to $96.0^{\circ}$ while the $\mathrm{C} 2-\mathrm{B} 14-\mathrm{C} 3$ chain along [111] bends from $173.3^{\circ}$ to $152.1^{\circ}$ to accommodate the existence of Fe atom. After shear strain increases to 0.191 , the B118-B111-B105 angle increases farther to $134.6^{\circ}$ by pulling the B118 atom out of the icosahedron, as shown in Fig. 5(b, e). For the C-B-C chain, the C2- 
B14-C3 angle bends more to $97.3^{\circ}$. The comparison of the C2-B14-C3 angle changes in GB-I and Fe-doped GB models are summarized in Fig. S1(a). The B164 atom originally belonging to the adjacent icosahedron is dragged closer to B105. The distance between B164 and B105 decreases significantly from 5.931 to $1.958 \AA$, while the B164-B166 distance increases from 2.219 to $5.013 \AA$. However, the B166-B155 bond distance remains $2.017 \AA$. Further increasing the shear strain to 0.209 leads to deconstruction of one icosahedra layer (Grain B) within the GB1 region, as shown in Fig. 5(c,f). In particular, the widely separated B164 and B116 (3.806 $\AA$ ) from two adjacent icosahedra forms a new bond $(1.663 \AA)$ in the GB region. Meanwhile, similar to the GB-I model, the distance between B166 and B155 increases to $4.068 \AA$. The distance comparison of B166-B155 in GB-I and Fe-doped GB models are summarized in Fig. S1(b). B118, B111 and B105 diffuse randomly in the GB region. During the shear process, the icosahedra in the GB2 region do not deconstruct, which is similar to the GB-I model. The failure mechanisms of the Fe-doped GB involve the interaction of icosahedra with impurity Fe atoms, leading to deconstruction of the icosahedra within the GB region. Balakrishnarajan et al. ${ }^{49}$ examined the stability effect of adding or removing an electron from the icosahedron. Our simulations show that the addition of electrons from Fe atoms weakens the icosahedron, which can explain why the existence of impurity $(\mathrm{Fe})$ further weakens the strength of the $\mathrm{B}_{4} \mathrm{C}$ GBs. 
(a)

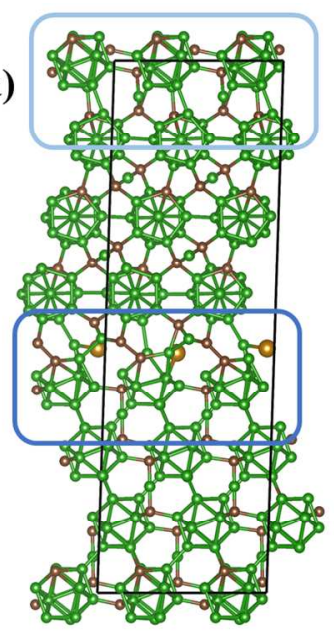

Strain $=0$

(d)

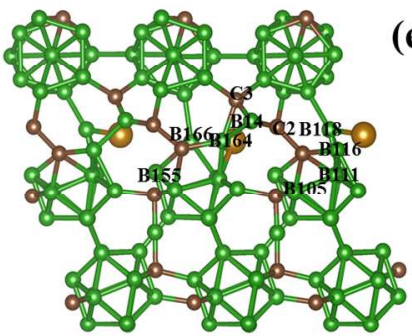

Strain $=0$

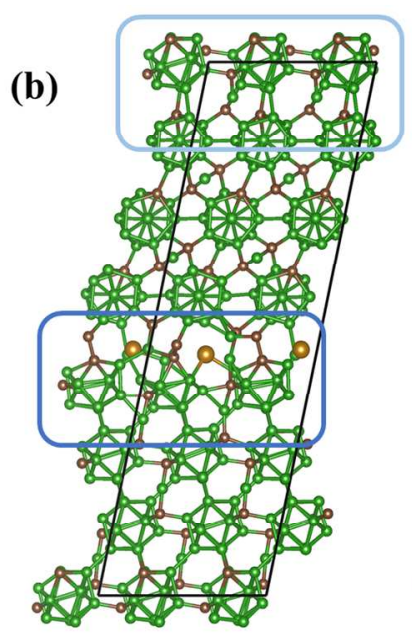

Strain $=0.191$

e)

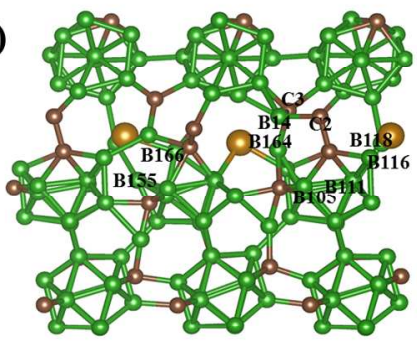

Strain $=0.191$

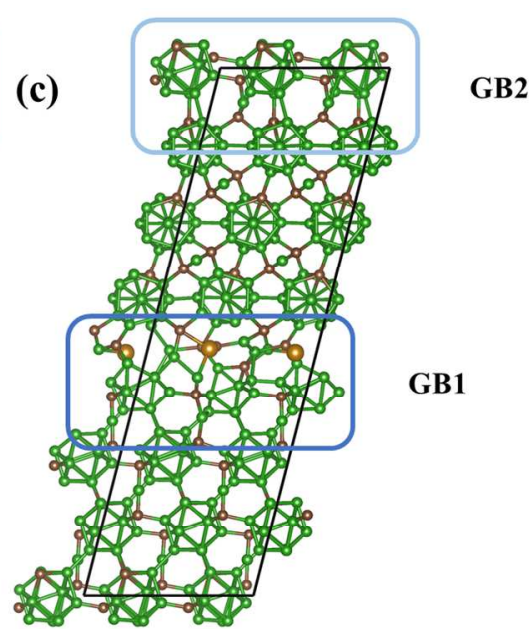

GB2

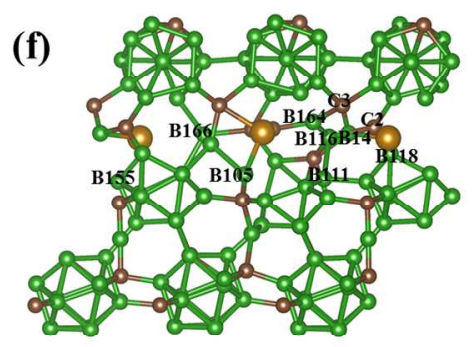

Strain $=0.209$

Figure 5. The structures evolution of Fe-doped GB model under pure shear deformation: (a,d) the intact structure; (b,e) the structure at 0.191 strain corresponding to the maximum shear stress; (c,f) the failed structure at 0.209 strain. The boron and carbon atoms are represented by the green and sienna balls, respectively. The Fe atom is represented by the yellow ball.

\subsubsection{Deformation mechanisms under biaxial shear deformation}

Our predictions on the mechanical response of the $\mathrm{B}_{4} \mathrm{C}$ GBs can be validated through indentation experiments; however, the stress conditions under indentation experiments are very complex compared to the pure shear deformation that we simulated. Thus, to predict the GB behavior and the impurity effects under indentation experiments, we performed biaxial shear deformation on the GB-I and the Fe-doped GB models. The shear stress-strain relationships for both GB models are shown in Fig. 6. For the GB-I model, the shear stress first increases to 20.2 $\mathrm{GPa}$ at 0.136 strain, then it decreases to $18.9 \mathrm{GPa}$ at 0.155 strain, and further increases to a maximum shear stress of $22.7 \mathrm{GPa}$ at 0.209 strain. Finally, GB-I model fails at 0.227 strain with 
the shear stress releasing to $17.6 \mathrm{GPa}$. The critical shear stress of the GB $(22.7 \mathrm{GPa})$ is lower than that of single crystal $\mathrm{B}_{4} \mathrm{C}(28.5 \mathrm{GPa})$ under indentation stress conditions from our previous simulations ${ }^{10}$ indicating that the presence of GBs decrease the strength of $\mathrm{B}_{4} \mathrm{C}$. This is consistent with the pure shear deformation simulations.

Compared to the GB-I model, the addition of Fe atom into the GB reduces slightly the maximum shear stress to $22.2 \mathrm{GPa}$, suggesting that the impurity has little effects on the indentation strength of the $\mathrm{B}_{4} \mathrm{C}$ interface. However, the critical failure strain for Fe-doped GB model is 0.173 which is $17.2 \%$ smaller than that of GB-I model $(0.209)$. The predicted critical shear stress ( $\sim 22 \mathrm{GPa})$ for GB models under biaxial shear stress can be compared with the indentation experiments on nanocrystalline $\mathrm{B}_{4} \mathrm{C}$ to validate our prediction.

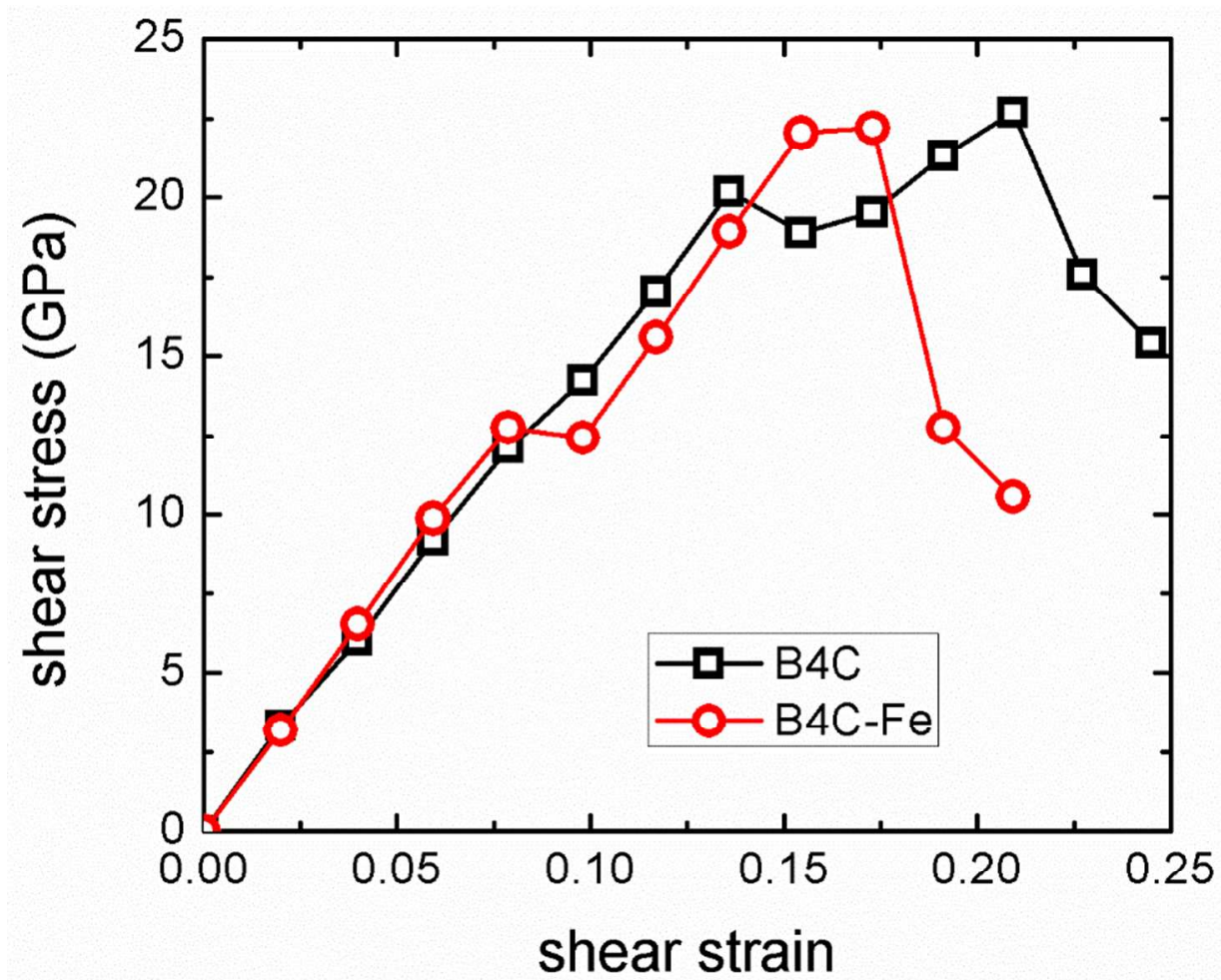

Figure 6. Shear-stress-shear-strain relationship of GB-I model (black $\square$ ) and Fe-doped GB model (red $\circ$ ) by shearing along GB under indentation stress conditions. 
To understand the failure mechanisms of the $\mathrm{B}_{4} \mathrm{C}$ GB under indentation stress conditions, the detailed deformation processes of GB-I model at various strain states are displayed in Fig. 7. Because of the high compressive stress conditions under biaxial shear loading, both GB1 and GB2 experience failure mechanisms different from that of pure shear deformation (in Fig. 3). The 0 strain GB-I structure is displayed in Fig. 7(a). As the shear strain increases to 0.136 corresponding to the first maximum shear stress, the icosahedra in GB1 region distort severely due to the interaction between C-B-C chains and icosahedra, as shown in Fig. 7(b). As the shear strain increases farther to 0.155 (shown in Fig. 7(c)), the icosahedra within GB1 start to deconstruct, while all icosahedra in GB2 remain intact. Thus, the first drop of shear stress in Fig.6 is ascribed to deconstruction of icosahedra within the GB1 region arising from interaction of C-B-C chains with icosahedra. As the shear strain increases to 0.209 , corresponding to the maximum shear stress, the deconstructed icosahedral layer within the GB1 region starts to collapse into the nearby icosahedral layer in Grain B, as shown in Fig. 7(d). But the icosahedra within GB2 region are not yet deconstructed. At 0.277 strain, the icosahedra in the GB2 region have disintegrated (Fig. 7(e)), leading to the shear stress release and mechanical failure. 


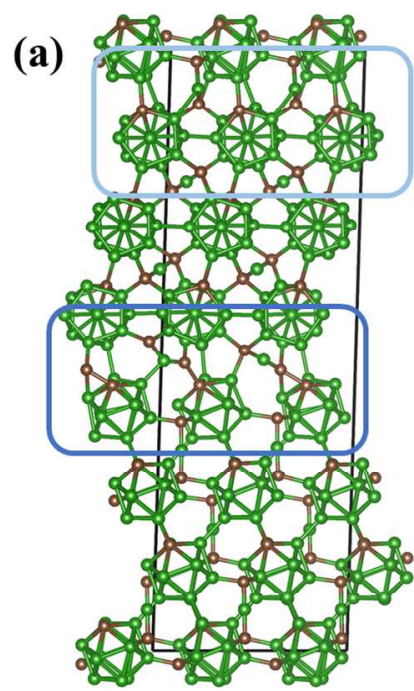

Strain $=0$

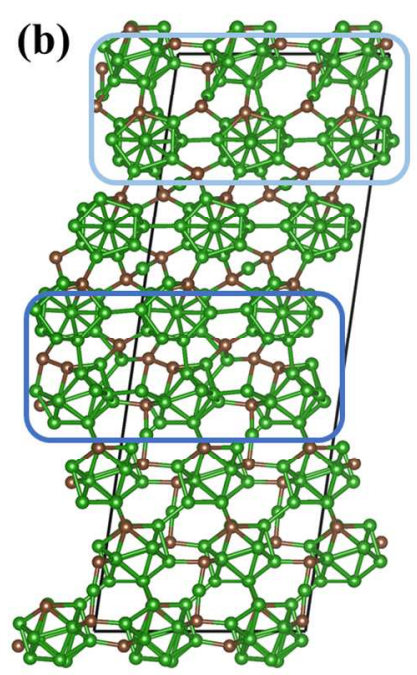

Strain $=\mathbf{0 . 1 3 6}$

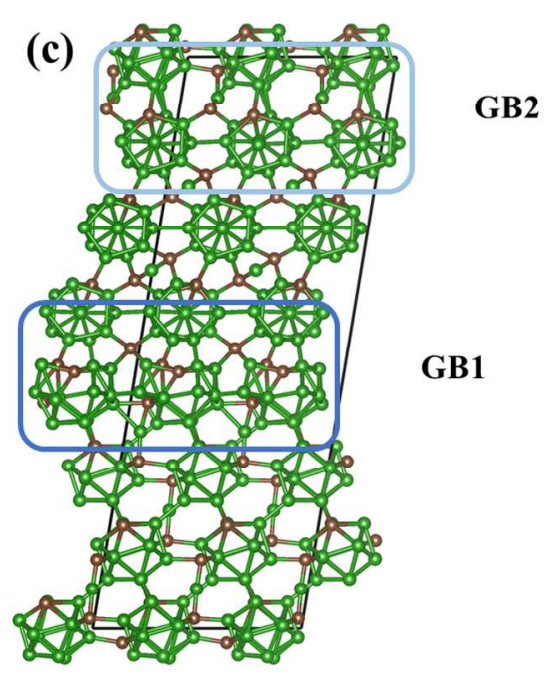

Strain $=\mathbf{0 . 1 5 5}$

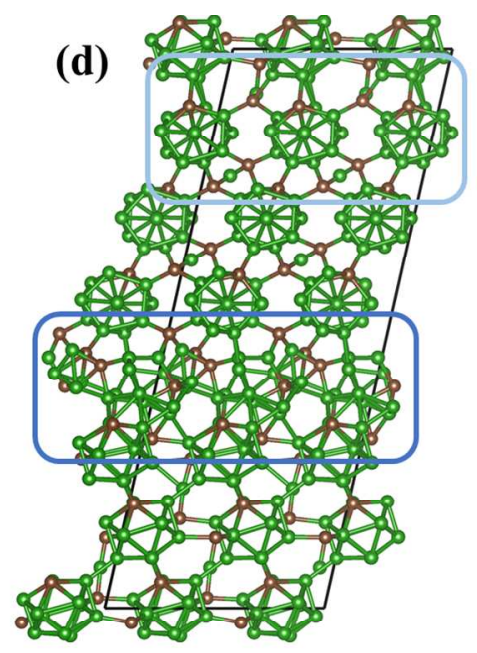

Strain=0.209

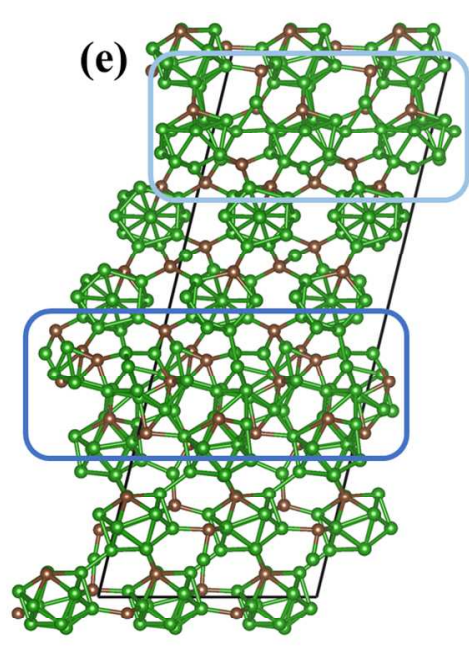

Strain $=\mathbf{0 . 2 2 7}$

Figure 7. The structural evolution of the GB-I model under biaxial shear deformation: (a) the intact structure; (b) the structure at 0.136 strain; (c) the structure at 0.155 strain corresponding to the first stress drop; (d) the structure at 0.209 strain corresponding to the maximum shear stress; (e) the failed structure at 0.227 strain. The boron and carbon atoms are represented by the green and sienna balls, respectively.

To understand the deformation processes, the GB1 region is enlarged in Fig. S2. The 0 strain GB1 structure is displayed in Fig. S2(a). At 0.136 shear strain (Fig. S2(b)), the B155 boron atom is dragged out from the icosahedral cage, while the C39-B155 bond distance increase from 1.687 $\AA$ at 0 strain to $2.193 \AA$. Meanwhile, the C2-B14-C3 chain bends from $173.3^{\circ}$ to $125.3^{\circ}$. The 
initial C-B-C chain bend is accommodating the mismatch of GBs. When the strain increases further to 0.155 (Fig. S2(c)), the shear stress is released by $1.3 \mathrm{GPa}$. The distance between chain boron (B7) and cage carbon (C39) decreases from 2.446 to $1.594 \AA$. The icosahedra further deconstruct by dragging B163 atom out of the cage. The B162-B163 bond distance increases from $1.856 \AA$ to $2.232 \AA$ as the shear strain increases to 0.209 (Fig. S2(d)) and increase further to $2.388 \AA$ at 0.227 strain (Fig. S2(e)). Two layers of icosahedra are compressed and deconstruct due to the highly compressive stress state at 0.227 strain. Therefore, the failure mechanism for GB1 is directly related to the compressive stress.

Different from pure shear deformation, the icosahedra in GB2 are deconstructed under biaxial shear deformation. The detailed deformation processes are displayed in Fig. S3. Fig. S3(a) displays the intact structure. The failure starts with bending the C23-B103-C26 chain angle from $143.5^{\circ}$ at 0.136 strain (Fig. S3(b)) to $139.1^{\circ}$ at 0.209 strain (Fig. S3(c)) which corresponds to the critical stress for GB-I model. Then the C23-B103-C26 chain angle further decreases to $126.8^{\circ}$ at 0.227 strain (Fig. S3(d)). However, the icosahedra in the GB2 region do not deconstruct until the shear stain increases to 0.227 . At 0.227 strain, the B40-B29 decreases from 1.833 to $1.753 \AA$. Therefore, the deconstruction of icosahedra in both GB1 and GB2 region leads to mechanical failure of GB-I model.

The deformation processes of Fe-doped GB model under biaxial shear deformation are displayed in Fig. 8. The structure at strain $=0$ is displayed in Fig. 8(a). As the shear strain increases to 0.173 , corresponding to the maximum shear stress, the icosahedra within both GB1 and GB2 region do not disintegrate, as shown in Fig. 8(b). However, as the shear strain increases farther to 0.193, the icosahedra deconstruct in both the GB1 and GB2 region (Fig. 8(c)), even though there is no Fe atom in the GB2 region. 


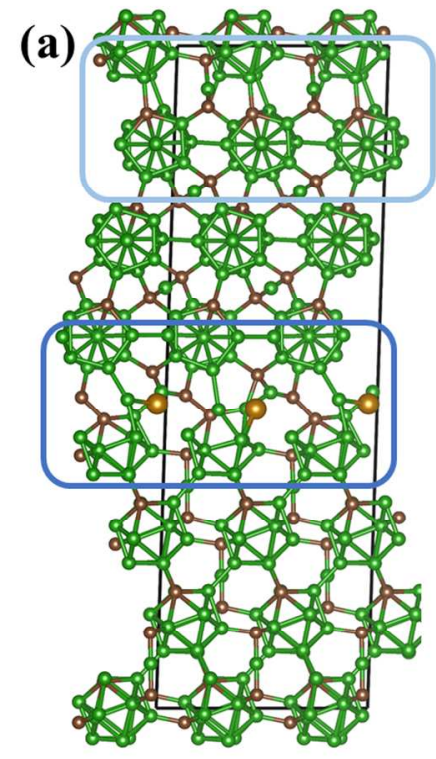

Strain $=0$

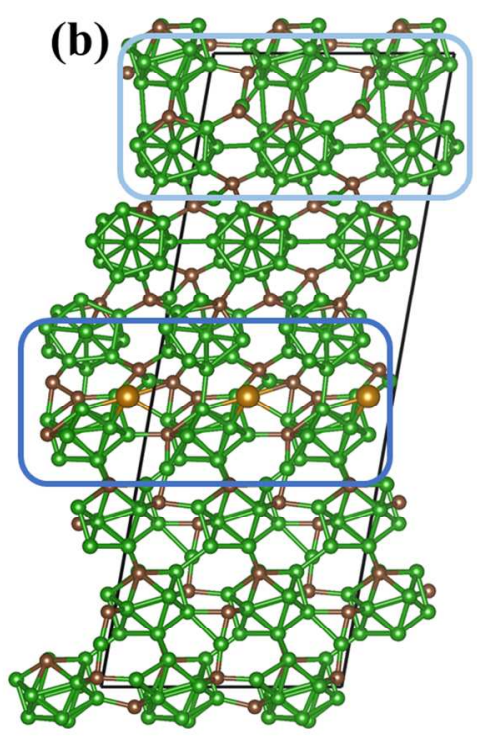

Strain $=\mathbf{0 . 1 7 3}$

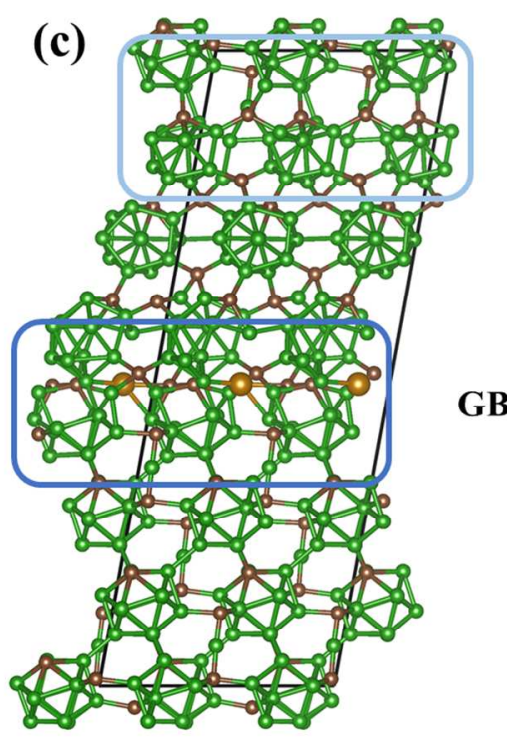

Strain $=\mathbf{0 . 1 9 1}$

Figure 8. The structures evolution of Fe-doped GB model under pure shear deformation: (a) the intact structure; (b) the structure at 0.173 strain corresponding to the maximum shear stress; (c) the failed structure at 0.191 strain. The boron and carbon atoms are represented by the green and sienna balls, respectively. The Fe atom is represented by the yellow ball.

To further examine the deformation process, we enlarged GB1 region of Fe-doped GB model while shearing under biaxial shear conditions at different strains, as shown in Fig. S4. The structure at strain $=0$ is displayed in Fig. S4(a). As the shear strain increases to $0.173, \mathrm{Fe}$ is pressed into the cage, while the Fe2-B159 distance increases from $1.830 \AA$ to $4.183 \AA$. As the shear strain increases to 0.191, the icosahedra on both sides of the Fe atom deconstruct (Fig. S4(c)). On the upper icosahedron, the B9-B13 bond breaks with the bond distance increasing from 1.891 to $2.893 \AA$; while on the lower icosahedron, the C39-B155 bond breaks with the bond distance increasing from 1.640 to $2.474 \AA$ A. Different from the GB-I model, the B162-B163 bond distance in Fe-doped GB model remains the same while increasing shear strain. The comparison of B162-B163 bond distances in GB-I and Fe-doped GB models are summarized in Fig. S5(a). In addition, the B166 atom from the lower icosahedron leaves the original cage and 
move upward to the upper icosahedron, decreasing the Fe2-B166 bond distance from 2.269 to $1.811 \AA$. Thus, the interaction of the Fe atoms with the icosahedra on both sides of the GB leads to deconstruction of icosahedra in GB1 region. Therefore, under biaxial shear conditions the amorphous band and mechanical failure likely initiates from the Fe doped GB region.

The detailed failure process within the GB2 of Fe-B ${ }_{4} \mathrm{C}$ GB model is displayed in Fig. S6. No icosahedra disintegrate at 0 and 0.173 strain, as shown in Fig. S6(a,b). The angle of C23-B103C26 chain decreases from $145.4^{\circ}$ at 0 strain to $119.9^{\circ}$ at 0.173 strain. As the shear strain increases to 0.191 , the C23-B103-C26 angle decreases farther to $115.2^{\circ}$ as shown in Fig. S6(c). The comparison of the C23-B103-C26 angle changes in GB-I and Fe-doped GB models are summarized in Fig. S5(b). Meanwhile, the B40-B35 icosahedral bond breaks and B40 forms a new B40-B43 bond with the B43 atom from the C-B-C chain, which leads to deconstruction of the icosahedron. Therefore, the failure of GB2 in Fe-doped GB can be ascribed to the interactions between C-B-C chain and icosahedra.

\section{Conclusions}

In summary, we constructed two generic $\mathrm{GB}$ models of $\mathrm{B}_{4} \mathrm{C}$ with different interfacial energies and applied QM simulations to elucidate their failure mechanisms. We also examined how Fe impurity affects the strength and failure mechanisms of one GB model. We found that the GB models have much lower critical shear strength than the single crystalline $\mathrm{B}_{4} \mathrm{C}$, suggesting the mechanical failure would initiate from GB regions in polycrystalline $\mathrm{B}_{4} \mathrm{C}$. The higher interfacial energy of GB-II model leads to lower strength but much higher critical failure strain, which suggests that to improve ductility it is critical to design high energy $\mathrm{GBs}$ in $\mathrm{B}_{4} \mathrm{C}$. The presence of Fe impurities further lowers the strength of $\mathrm{B}_{4} \mathrm{C}$ but does not increase ductility. 
The failure mechanisms of GB models are examined under both pure shear and biaxial shear deformation mimicking indentation, which reveals the following:

- Under pure shear deformation, the failure of the GB-I model arises from direct deconstruction of icosahedra in the GB1 region while no icosahedra disintegrate in the GB2 region. The presence of Fe impurities changes the failure mechanisms, which in this case arise from deconstruction of icosahedra due to interaction with the impurity atoms within the GB region.

- Under biaxial shear conditions for GB-I model, the icosahedra deconstruct in both the GB1 and GB2 region because of the highly compressive stress. In the Fe-doped GB model the interaction of $\mathrm{Fe}$ atoms with the icosahedra facilitate deconstruction of icosahedra in theGB1 region. In the Fe-doped GB model, this also deconstructs icosahedra within the GB2 region at a lower shear strain of 0.191 even though there is no Fe atom dopant in the GB2 region.

\section{ASSOCIATED CONTENT}

\section{Supporting Information}

The Supporting Information (SI) is available free of charge on the ACS Publications website. The SI includes the comparison of (a) the angles of C2-B14-C3 chain, and (b) the B166 and B155 bond distances for both the GB-I and Fe-doped GB models under pure shear conditions as a function of shear strains; The GB1 structural evolution of GB-I model under biaxial shear deformation; The GB2 structural evolution of the GB-I model under biaxial shear deformation: (a) the intact structure; The GB1 structural evolution of the Fe-doped GB model under biaxial shear deformation; The comparison of (a) the B162-B163 bond distance and (b) the C23-B105C26 chain angle in GB-I and Fe-doped GB models under biaxial shear conditions at different 
shear strains; and the GB2 structural evolution of Fe-doped GB model under biaxial shear deformation.

\section{Acknowledgements}

This work is supported by the National Science Foundation (CMMI-1727428). SPC and JCL received the support from Army Research Laboratory under Cooperative Agreement Number W911NF-12-2-0022. WAG is supported by Defense Advanced Research Projects Agency (W31P4Q-13-1-0010 and W31P4Q1210008).

\section{Additional information}

The authors declare no competing financial interest.

\section{References}

(1) Shirai, K. Electronic Structures and Mechanical Properties of Boron and Boron-Rich Crystals (Part 2). J. Superhard Mater. 2010, 32, 336-345.

(2) Emin, D. Unusual Properties of Icosahedral Boron-Rich Solids. J. Solid State Chem. 2006, $179,2791-2798$.

(3) Yan, X. Q.; Tang, Z.; Zhang, L.; Guo, J. J.; Jin, C. Q.; Zhang, Y.; Goto, T.; McCauley, J. W.; Chen, M. W. Depressurization Amorphization of Single-Crystal Boron Carbide. Phys. Rev. Lett. 2009, 102, 75505.

(4) Fanchini, G.; Mccauley, J. W.; Chhowalla, M. Behavior of Disordered Boron Carbide under Stress. Phys. Rev. Lett. 2006, 97, 35502.

(5) Chen, M. W.; McCauley, J. W.; Hemker, K. J. Shock-Induced Localized Amorphization in Boron Carbide. Science 2003, 299, 1563-1566. 
(6) Chen, M. W.; McCauley, J. W.; LaSalvia, J. C.; Hemker, K. J. Microstructural Characterization of Commercial Hot-Pressed Boron Carbide Ceramics. J. Am. Ceram. Soc. 2005, $88,1935-1942$.

(7) Sezer, A. O; Brand, J. I. Chemical Vapor Deposition of Boron Carbide. Mater. Sci. Eng. 2001, B79, 191-202.

(8) Domnich, V.; Reynaud, S.; Haber, R. A.; Chhowalla, M. Boron Carbide: Structure, Properties, and Stability under Stress. J. Am. Ceram. Soc. 2011, 94, 3605-3628.

(9) An, Q.; Goddard III, W. A. Microalloying Boron Carbide with Silicon to Achieve Dramatically Improved Ductility. J. Phys. Chem. Lett. 2014, 5, 4169-4174.

(10) An, Q.; Goddard III, W. A.; Cheng, T. Atomistic Explanation of Shear-Induced Amorphous Band Formation in Boron Carbide. Phys. Rev. Lett. 2014, 113, 95501.

(11) Reddy, K. M.; Liu, P.; Hirata, A.; Fujita, T.; Chen, M. W. Atomic Structure of Amorphous Shear Bands in Boron Carbide. Nat. Commun. 2013, 4, 2483.

(12) Chen, M.; McCauley, J. W. Mechanical Scratching Induced Phase Transitions and Reactions of Boron Carbide. J. Appl. Phys. 2006, 100, 123517.

(13) Yan, X. Q.; Li, W. J.; Goto, T.; Chen, M. W. Raman Spectroscopy of Pressure-Induced Amorphous Boron Carbide. Appl. Phys. Lett. 2006, 88, 2004-2007.

(14) Domnich, V.; Gogotsi, Y.; Trenary, M.; Tanaka, T. Nanoindentation and Raman Spectroscopy Studies of Boron Carbide Single Crystals. Appl. Phys. Lett. 2002, 81, 37833785.

(15) Lasalvia, J. C.; Campbell, J.; Swab, J. J.; McCauley, J. W. Beyond Hardness: Ceramics 
and Ceramic-Based Composites for Protection. Jom 2010, 62, 16-23.

(16) Hayun, S.; Kalabukhov, S.; Ezersky, V.; Dariel, M. P.; Frage, N. Microstructural Characterization of Spark Plasma Sintered Boron Carbide Ceramics. Ceram. Int. 2010, 36, $451-457$.

(17) Xie, K. Y.; Kuwelkar, K.; Haber, R. A.; LaSalvia, J. C.; Hemker, K. J.; Hay, R.

Microstructural Characterization of a Commercial Hot-Pressed Boron Carbide Armor Plate. J. Am. Ceram. Soc. 2016, 99, 2834-2841.

(18) Chen, M. W.; McCauley, J. W.; LaSalvia, J. C.; Hemker, K. J. Microstructural Characterization of Commercial Hot-Pressed Boron Carbide Ceramics. J. Am. Ceram. Soc. 2005, 88, 1935-1942.

(19) Behler, K. D.; Marvel, C. J.; LaSalvia, J. C.; Walck, S. D.; Harmer, M. P. Observations of Grain Boundary Chemistry Variations in a Boron Carbide Processed with Oxide Additives. Scr. Mater. 2018, 142, 106-110.

(20) An, Q.; Goddard III, W. A. Atomistic Origin of Brittle Failure of Boron Carbide from Large-Scale Reactive Dynamics Simulations: Suggestions toward Improved Ductility. Phys. Rev. Lett. 2015, 115, 105501.

(21) Wade, K. The Structural Significance of the Number of Skeletal Bonding Electron-Pairs in Carboranes, the Higher Boranes and Borane Anions, and Various Transition-Metal Carbonyl Cluster Compounds. J. Chem. Soc. D Chem. Commun. 1971, 15, 792-793.

(22) Becher, P. F.; Painter, G. S.; Shibata, N.; Waters, S. B.; Linz, H. T. Effects of Rare-Earth (RE) Intergranular Adsorption on the Phase Transformation, Microstructure Evolution, 
and Mechanical Properties in Silicon Nitride with RE 2O 3+MgO Additives: RE=La, Gd, and Lu. J. Am. Ceram. Soc. 2008, 91, 2328-2336.

(23) Da Chen; Zhang, X.-F.; Ritchie, R. O. Effects of Grain-Boundary Structure on the Strength, Toughness, and Cyclic-Fatigue Properties of a Monolithic Silicon Carbide. $J$. Am. Ceram. Soc. 2000, 83, 2079-2081.

(24) Lipp, A. Boron Carbide: Production, Propterties and Applications; Technische Rundschau, 1965, 57, [14,28,33].

(25) Telle, R. Boride and Carbide Ceramics. In Structure and Properties of Ceramics, Materials Science and Technology v11; VCH Publishers: New York, NY, 1994; pp 173266.

(26) Alan W. Weimer. Carbide, Nitride and Boride Materials Synthesis and Processing. Chapman \& Hall, New York 1997, pp 671-692.

(27) Suri, A. K.; Subramanian, C.; Sonber, J. K.; Murthy, T. S. R. C. Synthesis and Consolidation of Boron Carbide: A Review. Int. Mater. Rev. 2010, 55, 4-40.

(28) Champagne, B.; Angers, R. Mechanical Properties of Hot-Pressed B-B4C Materials. J. Am. Ceram. Soc. 1979, 62, 149-153.

(29) An, Q.; Goddard III, W. A.; Xie, K. Y.; Sim, G. D.; Hemker, K. J.; Munhollon, T.; Fatih Toksoy, M.; Haber, R. A. Superstrength through Nanotwinning. Nano Lett. 2016, 16, $7573-7579$.

(30) Kresse, G.; Hafner, J. Ab Initio Molecular Dynamics for Liquid Metals. Phys. Rev. B 1993, $47,558-561$. 
(31) Kresse, G.; Furthmüller, J. Efficient Iterative Schemes for Ab Initio Total-Energy Calculations Using a Plane-Wave Basis Set. Phys. Rev. B 1996, 54, 11169-11186.

(32) Kresse, G.; Furthmüller, J. Efficiency of Ab-Initio Total Energy Calculations for Metals and Semiconductors Using a Plane-Wave Basis Set. Comput. Mater. Sci. 1996, 6, 15-50.

(33) Perdew, J. P.; Burke, K.; Ernzerhof, M. Generalized Gradient Approximation Made Simple. Phys. Rev. Lett. 1996, 77, 3865-3868.

(34) Kresse, G. From Ultrasoft Pseudopotentials to the Projector Augmented-Wave Method. Phys. Rev. B 1999, 59, 1758-1775.

(35) Blöchl, P. E.; Jepsen, O.; Andersen, O. K. Improved Tetrahedron Method for BrillouinZone Integrations. Phys. Rev. B 1994, 49, 16223-16233.

(36) Methfessel, M.; Paxton, A. T. High-Precision Sampling for Brillouin-Zone Integration in Metals. Phys. Rev. B 1989, 40, 3616-3621.

(37) Roundy, D.; Krenn, C.; Cohen, M.; Morris, J. Ideal Shear Strengths of Fec Aluminum and Copper. Phys. Rev. Lett. 1999, 82, 2713-2716.

(38) Li, B.; Sun, H.; Chen, C. Large Indentation Strain-Stiffening in Nanotwinned Cubic Boron Nitride. Nat. Commun. 2014, 5, 4965.

(39) Zhou, X. F.; Oganov, A. R.; Shao, X.; Zhu, Q.; Wang, H. T. Unexpected Reconstruction of the $\alpha$-Boron (111) Surface. Phys. Rev. Lett. 2014, 113, 176101.

(40) Brostow, W.; Dussault, J. P.; Fox, B. L. Construction of Voronoi Polyhedra. J. Comput. Phys. 1978, 29, 81-92.

(41) Villars, P.; Prince, A.; Okamoto, H. Handbook of Ternary Phase Diagrams; Massalski, T. 
B., Ed.; ASM international: Metals Park, OH, 1997.

(42) An, Q.; Goddard III, W. A. Boron Suboxide and Boron Subphosphide Crystals: Hard Ceramics That Shear without Brittle Failure. Chem. Mater. 2015, 27, 2855-2860.

(43) An, Q. Prediction of Superstrong Tau-Boron Carbide Phase from Quantum Mechanics. Phys. Rev. B 2017, 95, 2-5.

(44) An, Q.; Goddard III, W. A.; Xiao, H.; Cheng, T. Deformation Induced Solid-Solid Phase Transitions in Gamma Boron. Chem. Mater. 2014, 26, 4289-4298.

(45) Tang, B.; An, Q.; Goddard III, W. A. Improved Ductility of Boron Carbide by Microalloying with Boron Suboxide. J. Phys. Chem. C 2015, 119, 24649-24656.

(46) Jiang, C.; Srinivasan, S. G. Unexpected Strain-Stiffening in Crystalline Solids. Nature 2013, 496, 339-342.

(47) Xie, K. Y.; Domnich, V.; Farbaniec, L.; Chen, B.; Kuwelkar, K.; Ma, L.; McCauley, J. W.; Haber, R. A.; Ramesh, K. T.; Chen, M.; Hemker, K. J. Microstructural Characterization of Boron-Rich Boron Carbide. Acta Mater. 2017, 136, 202-214.

(48) Madhav Reddy, K.; Guo, J. J.; Shinoda, Y.; Fujita, T.; Hirata, A.; Singh, J. P.; McCauley, J. W.; Chen, M. W. Enhanced Mechanical Properties of Nanocrystalline Boron Carbide by Nanoporosity and Interface Phases. Nat. Commun. 2012, 3, 1052.

(49) Balakrishnarajan, M. M.; Pancharatna, P. D.; Hoffmann, R. Structure and Bonding in Boron Carbide: The Invincibility of Imperfections. New J. Chem. 2007, 31, 473-485. 


\section{TOC Figure}
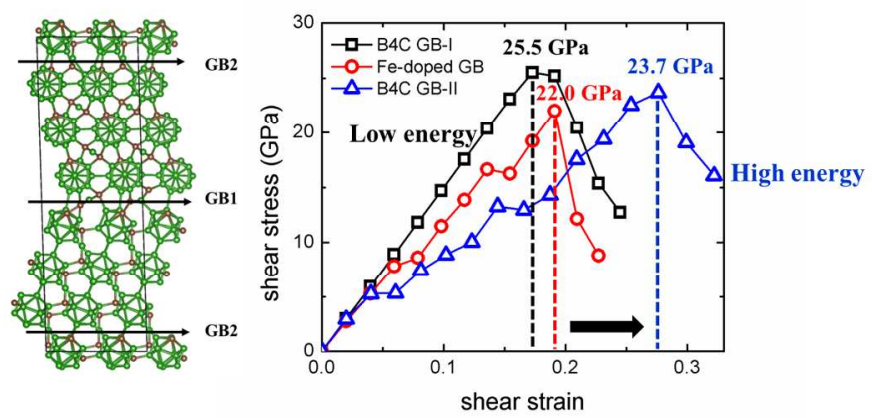

16

17

18

19

20

21

22

23

24

25

26

27

28

29

30

31

32

33

34

35

36

37

38

39

40

41

42

43

44

45

46

47

48

49

50

51

52

53

54

55

56

57

58

59

60 